PLANNING TOOLS FOR ESTIMATING RADIATION EXPOSURE AT THE NATIONAL IGNITION FACILITY

J. Verbeke, M. Young, S. Brereton, L. Dauffy, J. Hall, L. Hansen, H, Khater, S. Kim, B. Pohl, S. Sitaraman

October 28, 2010

19th Topical Meeting on the Technology of Fusion Energy Las Vegas, NV, United States November 7, 2010 through November 11, 2010 
This document was prepared as an account of work sponsored by an agency of the United States government. Neither the United States government nor Lawrence Livermore National Security, LLC, nor any of their employees makes any warranty, expressed or implied, or assumes any legal liability or responsibility for the accuracy, completeness, or usefulness of any information, apparatus, product, or process disclosed, or represents that its use would not infringe privately owned rights. Reference herein to any specific commercial product, process, or service by trade name, trademark, manufacturer, or otherwise does not necessarily constitute or imply its endorsement, recommendation, or favoring by the United States government or Lawrence Livermore National Security, LLC. The views and opinions of authors expressed herein do not necessarily state or reflect those of the United States government or Lawrence Livermore National Security, LLC, and shall not be used for advertising or product endorsement purposes. 


\title{
PLANNING TOOLS FOR ESTIMATING RADIATION EXPOSURE AT THE NATIONAL IGNITION FACILITY
}

\author{
LLNL-CONF-461262
}

\author{
J. Verbeke, M. Young, S. Brereton, L. Dauffy, J. Hall, L. Hansen, \\ H. Khater, S. Kim, B. Pohl, and S. Sitaraman \\ Lawrence Livermore National Laboratory, Livermore, 9000 East Avenue, Livermore, CA 94550 \\ verbeke2@llnl.gov
}

A set of computational tools was developed to help estimate and minimize potential radiation exposure to workers from material activation in the National Ignition Facility (NIF). AAMI (Automated ALARA-MCNP Interface) provides an efficient, automated mechanism to perform the series of calculations required to create dose rate maps for the entire facility with minimal manual user input. NEET (NIF Exposure Estimation Tool) is a web application that combines the information computed by AAMI with a given shot schedule to compute and display the dose rate maps as a function of time. AAMI and NEET are currently used as work planning tools to determine stay-out times for workers following a given shot or set of shots, and to help in estimating integrated doses associated with performing various maintenance activities inside the target bay. Dose rate maps of the target bay were generated following a low-yield $10^{16} \mathrm{D}-\mathrm{T}$ shot and will be presented in this paper.

\section{INTRODUCTION}

In a facility like the National Ignition Facility (NIF), where neutron fluxes are intermittently present, it is important to accurately analyze the ambient radiation exposure due to the activation of large number of surrounding materials and their subsequent decay, principally by gamma emission. Target Bay (TB) components, like target positioners (Cryo TARPOS and TARPOS), diagnostic instrument manipulators (DIMs), Final Optics Assemblies (FOAs) as well as utilities and surrounding structural materials are activated during yield shots. The present work is motivated by the need for the development of an automated mechanism that allows for simultaneous analysis of contribution resulting from all activated structures in any complex three-dimensional geometry to the radiation environment inside the NIF target bay. In order to provide an efficient method for estimating dose rates, a coupling scheme between radiation transport and neutron activation codes was developed. The approach used in developing this coupling scheme is somewhat similar to an approach used by the rigorous 2-step (R2S) computational method. ${ }^{1}$ A webbased application was also developed to use the calculated dose rates for estimating potential dose hazards associated with different maintenance activities following any given shot sequence, The estimated dose values will be compared to the actual dose measurements taken during the actual execution of the different maintenance tasks and the new tools allow for adjusting the predicted "calculated" dose values in the data base in accordance with the actual measured values.

\section{AAMI}

AAMI, which stands for Automated ALARA-MCNP Interface, ${ }^{2,3}$ automates the series of calculations required to create dose rate maps in the facility for a given neutron shot yield. The goal of this code is to produce data with minimal user input, henceforth minimizing human error. While the results shown here are all for the NIF facility, AAMI was designed with generality in mind and can thus be applied to any facility producing neutrons.

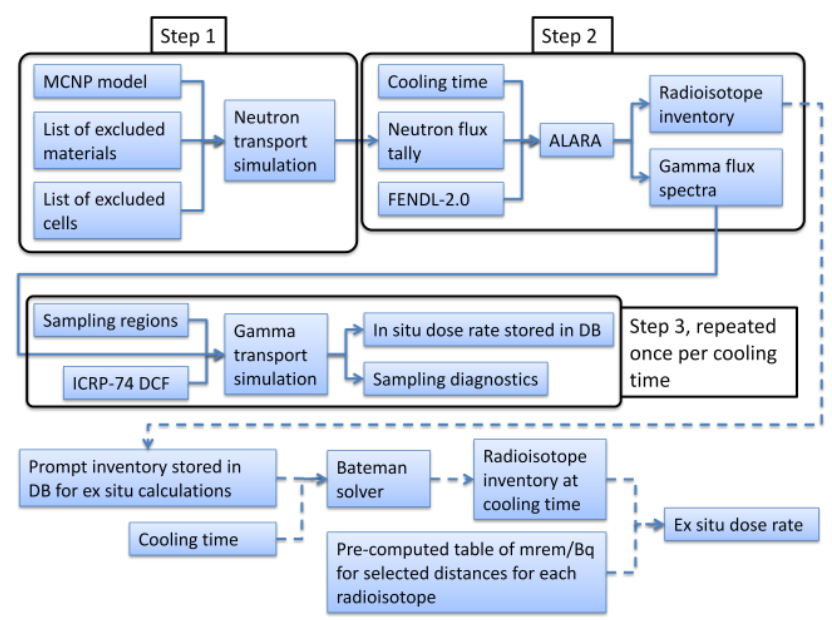

Fig. 1: Schematic flow chart showing how the dose rates are computed in AAMI.

The 3 steps of AAMI are summarized in Fig. 1. The first step consists of producing a MCNP (Ref. 4) model that contains all the components that are important from both radiation transport and material activation perspectives (see Fig. 2). From a radiation transport perspective, large concrete-like structures are important. The gunite layer around the target chamber for instance 
acts as a shield, lowering the prompt neutron population outside of the target chamber, and thus material activation. From a material activation perspective, it is important for the model to have structures (such as iron, aluminum) that become more activated by neutrons, as well as those structures that are inserted in the target chamber during the shots (see Fig. 3) and see a higher neutron flux. The latter structures are often retracted after a shot and represent a relatively more significant exposure source. In this first step, we determine all the components that can potentially be activated by specifying a list of MCNP cell numbers. A Monte Carlo continuous-energy simulation that emits D-T neutrons from the target is then carried out to tally the neutron flux spectra in all these components of interest. The source term for this run consisted of a point source with a realistic 101-group D-T neutron spectrum with a yield of $10^{16}$ neutrons placed at the center of the target chamber. The cross-section library used for the transport is FENDL-2.1.

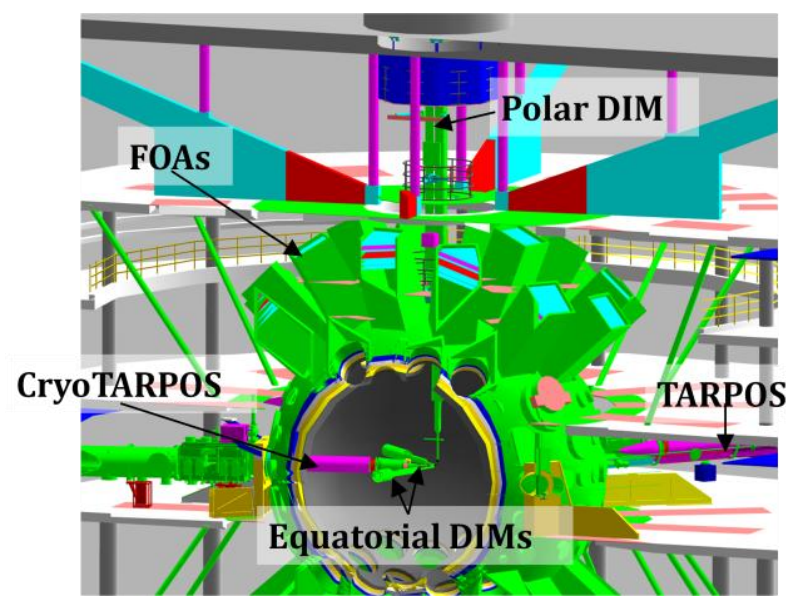

Fig. 2 Detailed model of the NIF facility used for radiation exposure calculations.

In the second step, AAMI takes the neutron flux spectrum computed for each MCNP cell and converts it into an inventory of radioisotopes and a $\gamma$-ray spectrum in a 25-group structure. The ALARA activation $\operatorname{code}^{6}$ and the FENDL-2.0 libraries ${ }^{7}$ are used to compute these inventories and $\gamma$-spectra, which are by nature of radioactivity time-dependent. Inventories of radioisotopes and $\gamma$-spectra for each MCNP cell are produced for the different cooling times specified by the user. The third step generates the dose rates from material activation in the entire NIF Target Bay. First, some activated components which are inserted into the target chamber during the shot for diagnostics are retracted outside of the target chamber. AAMI will work as long as the activated cell numbers and volumes do not change from the extended to the retracted model. The $\gamma$-rays will simply be emitted from the retracted location. Second, a fine 3- dimensional grid is imposed on the MCNP model of the NIF Target Bay for $\gamma$-rays tallying purposes.

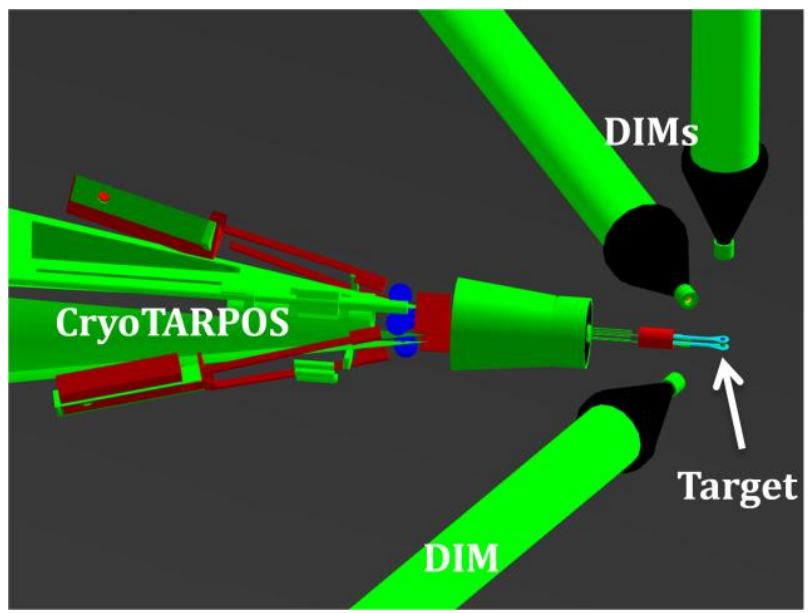

Fig. 3 Detailed model of the CryoTARPOS and 3 DIMs inserted into the target chamber during a shot.

In the third step, with a single simulation per cooling time, the $\gamma$-rays spectra computed in the second step are sampled and emitted from each activated component, and propagated by a transport simulation through the entire MCNP model of the NIF Target Bay. Of course, care was taken to account for the different $\gamma$-ray intensities in the different activated cells. The output file of MCNP contains a detailed diagnostic table showing how well or poorly the geometry was sampled by comparing the expected cell volumes with the cell volumes as calculated by $\gamma$-ray source sampling. From this simulation, we obtain a 3-dimensional $\gamma$-ray flux map, which MCNP converts into a dose rate map using the ICRP-74 fluence to effective dose conversion coefficients ${ }^{8}$ (see ICRP-74 Dose Conversion Factors in Fig. 1). Step 3 is repeated as many times as we have cooling times. The generated dose rate maps are stored in a database, along with the initial radionuclide inventories for activated components that may require handling.

The code MCNP was modified to simultaneously and efficiently sample the $\gamma$-rays from all activated components. The major modification consisted in a custom source subroutine, in which locations are first sampled within user-defined sampling regions (boxes, cylinders, cylinder wedges, tubes, tube wedges, spheres and sections thereof, shells and sections thereof, potentially transformed), then checked against a set of user-defined cells to see whether they belong to one of these cells, upon which the code samples the appropriate activation $\gamma$-ray distribution to emit a $\gamma$-ray from that location. The user-defined sampling regions can be assigned different importances. This powerful feature enables the user to bias the emission of activation $\gamma$-rays towards regions of higher activation. 
It is important to note that in producing the MCNP model, the model was designed considering the following needs: i) in transport, an adequate importance cell map is required for appropriate statistics; ii) in activation, the definition of cells for a spatial distribution of the residual $\gamma$ source should be good enough to perform subsequent accurate dose rate calculations. This definition of cells is not automated, since it is facility-dependent. The user experience will determine the best cell definition to fulfill both transport and activation needs. The applicability of AAMI to other facilities is possible but under these considerations.

\section{NEET}

The second computational tool, NEET (Ref. 9), stands for NIF Exposure Estimation Tool. It is a web application that can perform several types of radiation exposure calculations needed by health physicists for radiation protection. Its first purpose is to compute the dose rates after a single shot or set of shots. A shot schedule is entered in a table and combined with the dose rate information stored in the database to compute and display in situ dose rate maps as a function of time. For cooling times that are not in the database, NEET generally

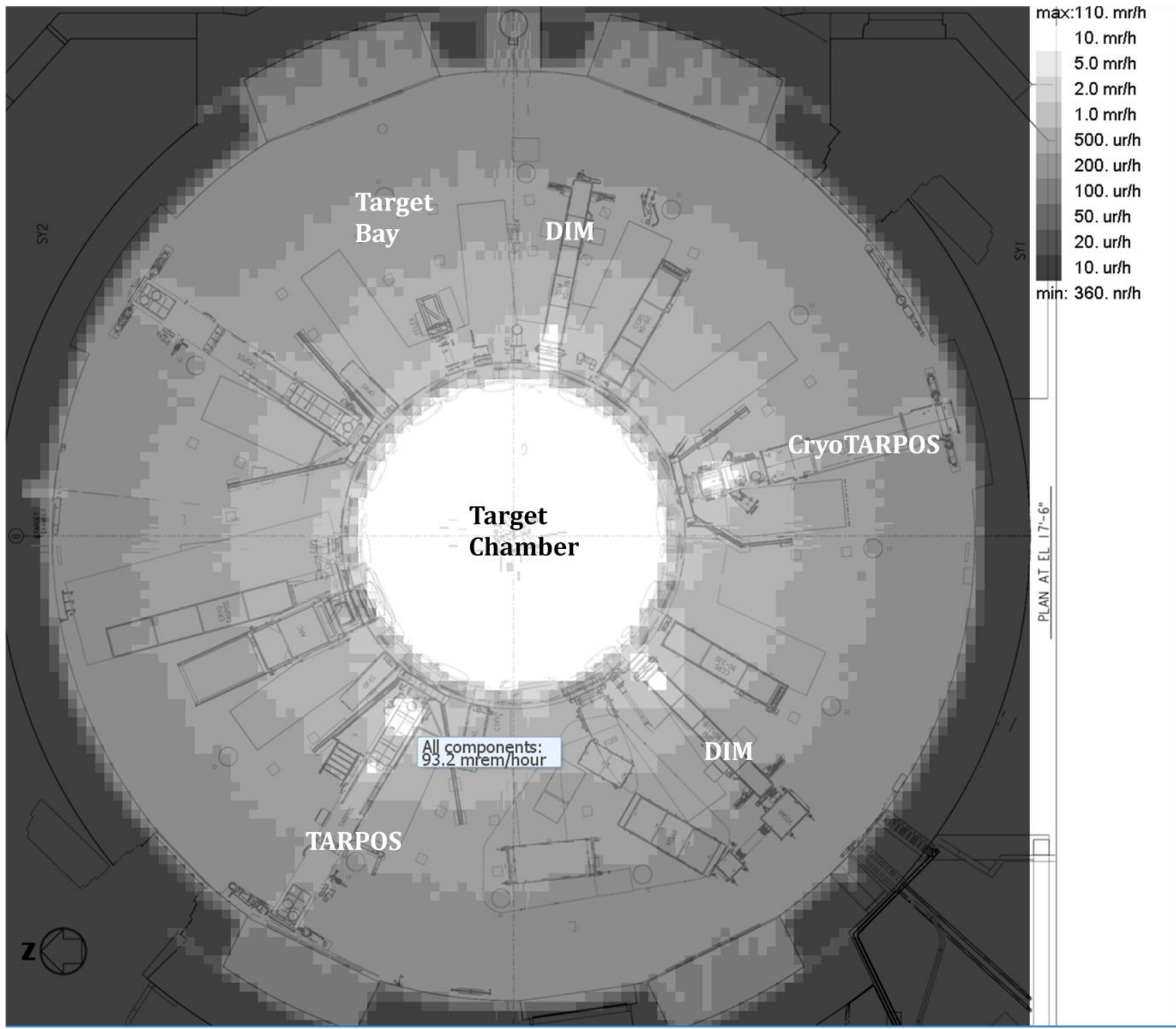

Fig. 4 In situ dose rate map at level 17'6" of the NIF building 1 hour after a single $10^{16}$ D-T neutron shot, a dose rate of 93.2 $\mathrm{mrem} / \mathrm{h}$ can be seen at the tip of the TARPOS. 
interpolates the dose rate data for stored cooling times. Fig. 4 shows the dose rate 1 hour following a single $10^{16}$ D-T neutron shot in the 17'6" floor level. As expected, the highest contact dose rates (in white) are at the tips of the retracted CryoTARPOS (140 mrem/h), TARPOS (93 $\mathrm{mrem} / \mathrm{h}$ ), and the two horizontal DIMs (102 and 109 $\mathrm{mrem} / \mathrm{h}$ ), because they are close to the target during the shot. Dark regions indicate low dose rates. By moving the cursor around the map, the local dose rates can be read. This map proves to be useful for planning purposes. By quickly glancing over it for different cooling times, one can determine at what time after a series of shots it is safe to reenter the TB from a radiological perspective. The user can click on any point on the map to see a graph of the dose rate as a function of time. Fig. 5 shows the dose rate at the tip of the TARPOS as a function of time for three $10^{16}$ D-T shots within 2 months. Because the DIMs are holding instruments that need to be handled after the shot, it is important to determine from this graph the time at which these instruments can be safely retrieved from a radiological perspective. The in situ dose rate calculated is the sum of the dose rates from the diagnostics instruments, manipulator and all surrounding activated components.

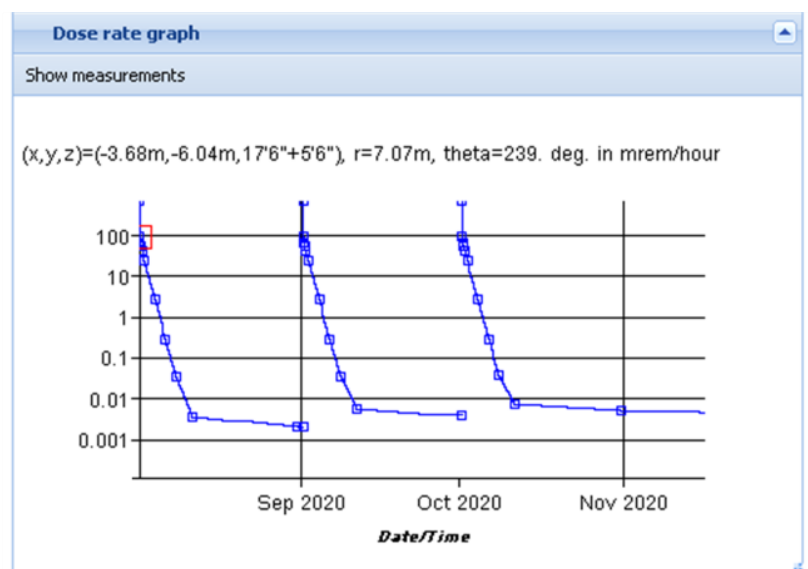

Fig. 5 In situ dose rate as function of time at the tip of the TARPOS. Box indicates 1 hour after the first $10^{16} \mathrm{D}$-T neutron shot.

A continuously updated list of dose rate measurements is stored in a database. The "Show measurements" button in Fig. 5 enables the user to overlay the dose rate measurements on the graph for a particular exposure location. If systematic discrepancies between measured and calculated dose rates are observed, the user has the option of using a correction factor to apply to the calculated dose rates. This option must be used with caution and should only be used once the discrepancies are fully understood.

Once an instrument has been safely retrieved from a manipulator and taken outside of the TB, one is no longer interested in computing the in situ dose rate, but the dose rate from the activated component itself ex situ. This can be done by using the ex situ capability of NEET, which is illustrated by steps 1,2 as well as the dashed lines in Fig. 1. NEET combines the initial radionuclide inventories stored in the database with the shot schedule entered by the user and then solves the Bateman equations ${ }^{10}$ to compute the radionuclide inventories in $\mathrm{Bq}$ as a function of time. It also uses a pre-computed table giving the dose rate per $\mathrm{Bq}$ as a function of the distance from the $\gamma$-ray source on a radionuclide by radionuclide basis. Combining this pre-computed table with the radionuclide inventory, the code computes for a given distance from the activated component the dose rate as a function of time after a set of shots for each radionuclide and adds them up to get the total ex situ dose rate, shown in Fig. 6.

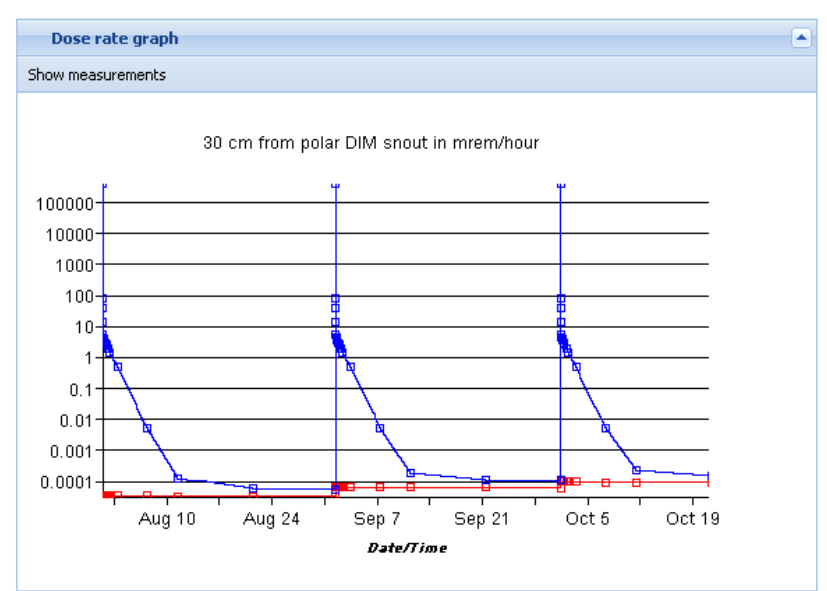

Fig. 6 Graph of total (upper curve) and ${ }^{54} \mathrm{Mn}$ (lower curve) ex situ dose rates $30 \mathrm{~cm}$ from the snout of the polar DIM, as a function of time. The shot schedule consisted of three $10^{16} \mathrm{D}-\mathrm{T}$ neutron shots on August 1, September 1 and October 1.

NEET also displays the list of the major nuclides contributing to the total dose rate and the user can select which one to display (lower curve in Fig. 6 is ${ }^{54} \mathrm{Mn}$ ). The graph in Fig. 6 is also shown in the tabulated form in Fig. 7 , where one can observe that ${ }^{54} \mathrm{Mn}$ contributes $0.6 \%$ of the total dose rate 6 days after the shot to become the major contributor with $55 \%$ of the total three weeks later.

Health physicists can also define maintenance activities composed of several sub-activities and calculate the integrated dose over the overall maintenance operation. As an example, the potential FOA maintenance shown in Fig. 8 consists of two sub-activities: replacing failing switch and tighten all screws. NEET integrates the dose rates over the periods of time for the two subactivities to determine the integrated doses per subactivity. Fig. 8 shows that the final optical assembly maintenance activities on September 29 resulted in integrated in situ doses of 211 and $237 \mu$ rem for the 2 sub-activities. NEET used a shot schedule consisting of 3 shots of yield $10^{16}$ D-T neutrons on Aug. 1, Sept. 1 and Oct. 1 for this calculation. Depending on the location of 
the maintenance activities, the health physicist can choose between the in situ and ex situ dose rates.

\begin{tabular}{|c|c|c|c|c|c|}
\hline \multicolumn{5}{|c|}{ Dose rates } & $\triangle$ \\
\hline Component & polar DIM & Location 3 & $30 \mathrm{~cm}$ from & in polar DIM sr $\vee$ & $S$ \\
\hline DaterTime & & Dose rate [mr & hrem/h] & Fraction of total & \\
\hline Sat Aug 01 & $202009: 59: 59 \mathrm{C}$ & & 0 & 0 & 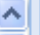 \\
\hline Sat Aug 01 & $202010: 00: 00 \mathrm{C}$ & & $34.26 e-6$ & $83.16 \mathrm{ppt}$ & \\
\hline Sat Aug 01 & $202010: 01: 00 \mathrm{C}$ & & $34.26 e-6$ & $405.1 \mathrm{ppb}$ & \\
\hline Sat Aug 01 & 2020 10:10:00 C & & $34.26 \mathrm{e}-6$ & $821.7 \mathrm{ppb}$ & \\
\hline Sat Aug 01 & $202010: 30: 00 \mathrm{C}$ & & $34.26 \mathrm{e}-6$ & $2.571 \mathrm{ppm}$ & \\
\hline Sat Aug 01 & $202011: 00: 00 \mathrm{C}$ & & $34.26 \mathrm{e}-6$ & $5.937 \mathrm{ppm}$ & \\
\hline Sat Aug 01 & $202012: 00: 00 \mathrm{C}$ & & $34.25 e-6$ & $7.523 \mathrm{ppm}$ & \\
\hline Sat Aug 01 & $202016: 00: 00 \mathrm{C}$ & & $34.24 e-6$ & $9.500 \mathrm{ppm}$ & \\
\hline Sat Aug 01 & $202022: 00: 00 \mathrm{C}$ & & $34.22 \mathrm{e}-6$ & $12.89 \mathrm{ppm}$ & \\
\hline Sun Aug 02 & $202010: 00: 00$ & & $34.18 e-6$ & $22.89 \mathrm{ppm}$ & \\
\hline Mon Aug 03 & $3202010: 00: 00$ & & $34.11 \mathrm{e}-6$ & $71.09 \mathrm{ppm}$ & \\
\hline Fri Aug 072 & $202010: 00: 00 \mathrm{G}$ & & 33.81e-6 & 6464. ppm & \\
\hline Tue Aug 11 & 2020 10:00:00 c & & 33.51e-6 & $26.31 \%$ & \\
\hline Fri Aug 212 & 2020 10:00:00 G & & $32.77 e-6$ & $55.05 \%$ & \\
\hline Tue Sep 01 & $202009: 59: 59$ ( & & $31.98 e-6$ & $56.73 \%$ & \\
\hline Tue Sep 01 & $202010: 00: 00$ & & $66.24 \mathrm{e}-6$ & $160.8 \mathrm{ppt}$ & \\
\hline Tue Sep 01 & $202010: 01: 00$ c & & $66.24 e-6$ & $783.2 \mathrm{ppb}$ & \\
\hline Tue Sep 01 & $202010: 10: 00 c$ & & $66.24 \mathrm{e}-6$ & $1.589 \mathrm{ppm}$ & 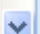 \\
\hline Compute & All components & $s$ & $m \sqcap-5$ & $\mathbf{v}$ & \\
\hline
\end{tabular}

Fig. 7 Table of ${ }^{54} \mathrm{Mn}$ ex situ dose rate $30 \mathrm{~cm}$ from the snout of the polar DIM, as a function of time. The shot schedule consisted of three $10^{16}$ D-T neutron shots on August 1, September 1 and October 1 (not shown). 'Fraction of total' in the $3^{\text {rd }}$ column refers to the ratio of ${ }^{54} \mathrm{Mn}$ to total ex situ dose rates.

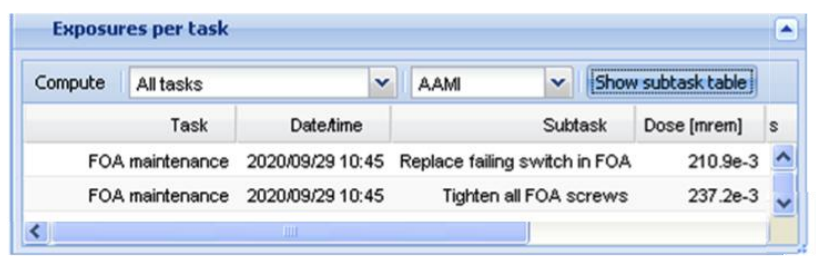

Fig. 8 Integrated in situ doses for possible maintenance activities.

\section{CONCLUSIONS}

The Radiation Safety Analysis Group at NIF has been developing two new tools to analyze the radiation exposure to personnel. AAMI and NEET are new work planning tools that are used together to effectively and with little human intervention to estimate stay-out times for workers following a set of shots. The tools can also be used to estimate integrated doses associated with performing various maintenance activities inside the target bay. The estimated dose values will be compared to the actual dose measurements taken during the actual execution of the different maintenance tasks and the new tools allow for adjusting the predicted "calculated" dose values in the data base in accordance with the actual measured values.

\section{ACKNOWLEDGMENTS}

This work performed under the auspices of the U.S. Department of Energy by Lawrence Livermore National Laboratory under Contract DE-AC52-07NA27344.

\section{REFERENCES}

1. Y. CHEN and U. FISCHER, "Rigorous MCNP Based Shutdown Dose Rate Calculations: Computational Scheme, Verification Calculations and Application to ITER," Fusion Engineering and Design 63-64, p.107-114 (2002).

2. M. YOUNG and J. VERBEKE, "AAMI: Automated ALARA-MCNP Interface User's Manual," LLNLPRES-426583, Lawrence Livermore National Laboratory (2010).

3. "Automated ALARA-MCNP Interface (AAMI) User's Manual," NIF-0116619, Lawrence Livermore National Laboratory (2010).

4. X-5 Monte Carlo Team, "MCNP - A General Monte Carlo N-Particle Transport Code, Version 5," LAUR-03-1987, Los Alamos National Laboratory (2003).

5. D. L. ALDAMA and A. TRKOV, "FENDL-2.1: Evaluated Nuclear Data Library for Fusion Applications," International Atomic Energy Agency, INDC(NDS)-467 (2004).

6. P. WILSON and D. HENDERSON, "ALARA: Analytic and Laplacian Adaptive Radioactivity Analysis," UWFDM-1070, University of Wisconsin Fusion Technology Institute, Madison, WI (1998).

7. A. PASHCHENKO et al., "FENDL/A-2.0: Neutron Activation Cross-Section Data Library for Fusion Applications," Report INDC (NDS)-173, IAEA Nuclear Data Section, March 1997.

8. International Commission on Radiological Protection, "Conversion Coefficients for use in Radiological Protection against External Radiation," ICRP Publication 74, Ann. ICRP26, Pergamon Press (1996).

9. "NEET: NIF Exposure Estimation Tool," NIF0116620, Lawrence Livermore National Laboratory (2010).

10. H. BATEMAN, "Partial Differential Equations of Mathematical Physics," Cambridge University Press (1932). 